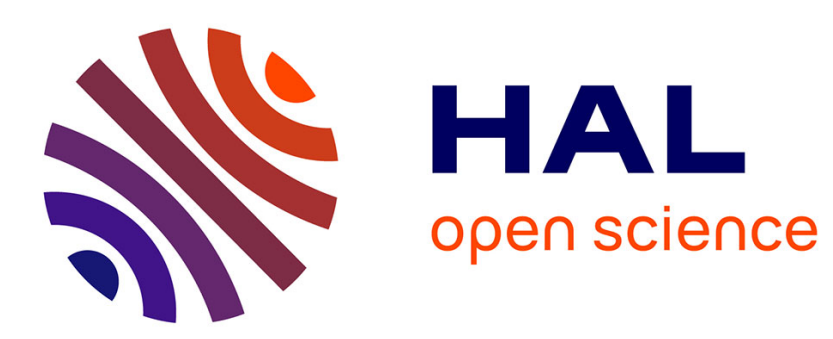

\title{
On Carrier QPSK Signal Detector Based on Second Order Cyclic-Moments
}

Nicolas Grollier, Sébastien Houcke

\section{To cite this version:}

Nicolas Grollier, Sébastien Houcke. On Carrier QPSK Signal Detector Based on Second Order CyclicMoments. WCNC 2018: IEEE Wireless Communications and Networking Conference, Apr 2018, Barcelone, Spain. 10.1109/WCNC.2018.8377024 . hal-01813235

\section{HAL Id: hal-01813235 https://hal.science/hal-01813235}

Submitted on 12 Jun 2018

HAL is a multi-disciplinary open access archive for the deposit and dissemination of scientific research documents, whether they are published or not. The documents may come from teaching and research institutions in France or abroad, or from public or private research centers.
L'archive ouverte pluridisciplinaire HAL, est destinée au dépôt et à la diffusion de documents scientifiques de niveau recherche, publiés ou non, émanant des établissements d'enseignement et de recherche français ou étrangers, des laboratoires publics ou privés. 


\title{
On Carrier QPSK Signal Detector Based on Second Order Cyclic-Moments
}

\author{
Nicolas Grollier, Sébastien Houcke \\ Institut Mines-Telecom, IMT Atlantique, Lab-STICC, UMR CNRS 6285, Univ-Bretagne Loire, 29238 Brest, France \\ Email: Firstname.Lastname@imt-atlantique.fr
}

\begin{abstract}
This paper considers the problem of detecting a QPSK communication signal on carrier. We propose a method to perform the detection thanks to second order cyclostationarity theory. It is well known that for such a signal, there is no cyclic frequency multiple of the studied signal carrier frequency. Hence, the basic method of second order cyclic-moments energy measurement is unable to detect any energy directly linked to the signal carrier. In this article, we propose a novel criterion based on second order cyclic-moments that exploits the convergence speed of the cyclic autocorrelation function estimator. We show that for a modulated QPSK signal the cyclic-correlation converges to zero but not in the same way if the cyclic frequency is a multiple of the carrier frequency or not. We develop a statistical test and derive the asymptotic probability density function of the criterion to propose a detection threshold. System performance simulation results are then evaluated by Monte Carlo simulations and compared to the results of fourth order nonlinear transformation method.
\end{abstract}

\section{INTRODUCTION}

This work has been done in the context of the FITNESS project, which goal is to develop a multi-standards novel generation PMR (Professional Mobile Radio) receiver. This kind of receiver has to digitize a large bandwidth to be able to deal with the different PMR standards. The digitization is performed after down conversion to an intermediate frequency to solve some analog issues. One of the difficulty is to detect the presence of a modulated QPSK signal in the digitalized bandwidth in order to adapt the receiver parameters. This detection should be able to work at low SNR (Signal to Noise Ratio) and with short observation duration.

Let us define by $f_{c}$ the carrier frequency of the signal to detect after the down frequency conversion. In the paper, we propose an algorithm to detect the presence of a modulated QPSK signal at frequency $f_{c}$ in noise. We propose a criterion based on second-order cyclostationarity feature. This criterion may be also of some use for spectrum sensing in cognitive radio or, for blind system identification for electronic warfare. In our context, we assume that neither the signal power nor the noise power are known. Moreover, the only prior available information about the signal to detect are: its carrier frequency and its modulation type (i.e. QPSK).

In [1], the authors summed up several reliable techniques to determine the signal presence in the sensed bandwidth. However, energy detection, waveform based-sensing, or match filter can not be applied considering our lack of knowledge on the signal to detect. Indeed, an energy detector requires to know the noise power and is not adapted to detect a specific carrier among a set several channels. For a match filter approach, a known pattern is needed or at least the emission filter and transmission rate.

That makes the cyclostationarity approach a natural candidate for our considered context. Moreover, the cyclostationarity property offers relevant tools where conventional methods do not work. In particular, it drawn interest in the application domain of cognitive radios. Thanks to its properties, this theory is used in several applications such as signal detection [2], source separation [3], fading channel identification [4], or modulation type classification [5].

However, in those applications some signals are difficult to analyze. This is the case of phase modulated signals on carrier frequency. Contrary to BPSK signals, modulated QPSK signals does not exhibit cyclic frequencies depending on the carrier frequency. Detection and classification of such signals is possible, but often requires to use a nonlinear transformation as in [6], or higher order cyclic statistics, as developed in [7]. In this last document, the proposed method creates a DC component at a particular cyclic frequency. This transformation allows a good detection and classification of the chosen modulation, but is sensitive to noise power. We also notice that the created spectral line is located at $4 f_{c}$. This implies that the sampling frequency $f_{s}$ has to be greater than $f_{s} \geqslant 8 f_{c}$ in order to avoid aliasing effects. That makes the hardware implementation of this kind of algorithm quite difficult. In [8], it is also proposed to use sixth order cumulants, which provides very interesting performance. This performance has to be balanced by an expensive cost of operations and also requires a large number of symbols for detection $(\geqslant 1000)$. A second order cyclic-moments based approach is developed in [9] for modulation classification. Kim and al. use the particularity that there is no cyclic frequency in order to classify QPSK signals. But, this kind of approach can only be used for classification and not for detection.

In the following, the signal model, an analysis of the problem arisen by on carrier QPSK signal and second order moments, as well as our criterion, are described in section II. 
The statistical test and the theoretical detection threshold are presented in section III. In section IV, Monte Carlo simulations are run to estimate the performance of the proposed algorithm, and section $\mathrm{V}$ concludes the paper.

\section{PROBLEM FORMULATION}

\section{A. Considered model}

Let us define the base-band model of the considered communication signal where $T$ is the symbol period, $h(t)$ is a waveform low-pass filter and $a_{k}$ are the QPSK complex signal symbols:

$$
s(t)=s_{R}(t)+j s_{I}(t)=\sum_{k=1}^{N} a_{k} h(t-k T)
$$

$s_{R}(t)$ and $s_{I}(t)$ are the real and imaginary parts of the base-band signal $s(t)$. We point out that as $s(t)$ is a QPSK signal, its real and imaginary parts are assumed independent and identically distributed. Considering a stationary Additive White Gaussian Noise (AWGN) channel, the received signal $x(t)$, of a QPSK signal emitted at carrier frequency $f_{c}$ can be expressed as:

$$
x(t)=x_{R}\left(t-\Delta_{t}\right)-x_{I}\left(t-\Delta_{t}\right)+n(t)
$$

where

$$
\left\{\begin{array}{l}
x_{R}(t)=s_{R}(t) \cos \left(2 \pi f_{c} t\right), \\
x_{I}(t)=s_{I}(t) \sin \left(2 \pi f_{c} t\right)
\end{array}\right.
$$

and $n(t)$ is an additive white Gaussian noise of zero mean and variance $\sigma_{n}^{2}$. In the following parts we take the propagation delay $\Delta_{t}$ in eq.(2), equal zero. A short development shows that this hypothesis has absolutely no impact on the method and does not lead to a loss of generality.

\section{B. Cyclostationary basics}

In this section, we derive the cyclostationary analysis of eq.(1) and eq.(2). Cyclostationary signals have an autocorrelation function, defined in eq.(4), which is periodic in time, and consequently admits a Fourier decomposition.

$$
R_{s}(t, \tau)=E\left[s(t) s^{*}(t-\tau)\right]
$$

Here $E[$.$] is the expectation operator and (.)* is the complex$ conjugate operation. The Fourier series decomposition of $R_{s}(t, \tau)$ is given by:

$$
R_{s}(t, \tau)=\sum_{\alpha \in I_{s}} R_{s}^{\alpha}(\tau) e^{j 2 \pi \alpha t}
$$

Let us define by $I_{s}=\left\{\frac{k}{T}, k \in \mathbb{Z}\right\}$ the set of cyclic frequencies of $s(t)$ defined in eq.(1). The theoretical cyclic autocorrelation function (CAF) at a cyclic frequency $\alpha$ is defined as the Fourier transform of $R_{s}(t, \tau)$.

$$
R_{s}^{\alpha}(\tau)=\lim _{T_{\alpha} \rightarrow \infty} \frac{1}{T_{\alpha}} \int_{0}^{T_{\alpha}} R_{s}(t, \tau) e^{-j 2 \pi \alpha t} d t
$$

As detailed in [10], the signal $s(t)$ is considered cyclostationary and its cyclic frequencies are multiple of $1 / T$. Coefficients $R_{s}^{\alpha}(\tau)$ are the CAFs of $s(t)$. In practice, for $k>1$ the magnitude of the $R_{s}^{\alpha}(\tau)$ drops quickly and we often consider only the cyclic frequency $\alpha=1 / T$. In our context, we assume to know the set of cyclic-frequencies of the signal we wish to detect.

We can now derive the cyclostationary analysis for the signal $x(t)$ defined in eq.(2). Using eq.(6), one can easily show the following relation:

$$
R_{x}^{\alpha}(\tau)=R_{x_{R}}^{\alpha}(\tau)+R_{x_{I}}^{\alpha}(\tau)
$$

So, eq.(7) is the sum of the CAF of $x_{R}(t)$ and $x_{I}(t)$. The theoretical CAF of $x_{R}(t)$ is given by:

$$
R_{x_{R}}^{\alpha}(\tau)= \begin{cases}\frac{1}{2} \cos \left(2 \pi f_{c} \tau\right) R_{s_{R}}^{\alpha}(\tau) & \text { if } \alpha=0 \text { or } \pm \frac{1}{T} \\ \frac{1}{4} e^{ \pm j 2 \pi f_{c} \tau} R_{s_{R}}(\tau) & \text { if } \alpha= \pm 2 f_{c} \\ 0 & \text { otherwise }\end{cases}
$$

With $I_{x_{R}}=\left\{\left\{\frac{k}{T}, 2 k f_{c}\right\}, k \in \mathbb{Z}\right\}$ the set of cyclic frequencies of $x_{R}(t)$ defined in eq.(3). As $I_{x_{R}}$ contains elements that are not multiple to each other, the signal $x_{R}(t)$ is called almost cyclostationary (see [10] for more details). In that case, the expression eq.(5) is in reality a Fourier-Bohr series.

Note that the CAF expression of $x_{I}(t)$, leads to:

$$
R_{x_{I}}^{\alpha}(\tau)= \begin{cases}\frac{1}{2} \cos \left(2 \pi f_{c} \tau\right) R_{s_{I}}^{\alpha}(\tau) & \text { if } \alpha=0 \text { or } \pm \frac{1}{T}, \\ -\frac{1}{4} e^{ \pm j 2 \pi f_{c} \tau} R_{s_{I}}(\tau) & \text { if } \alpha= \pm 2 f_{c}, \\ 0 & \text { otherwise. }\end{cases}
$$

With $I_{x_{I}}=\left\{\left\{\frac{k}{T_{s}}, 2 k f_{c}\right\}, k \in \mathbb{Z}\right\}$ the set of cyclic frequencies of $x_{I}(t)$ defined in eq.(3). We underline that cyclic frequencies multiple of $f_{c}$ exists in eq.(8) and eq.(9). Indeed, for baseband QPSK signals, the constellation projection in the complex or real plan is consistent to BPSK modulation. Consequently, real and imaginary parts of baseband QPSK have cyclic frequencies multiple of the carrier frequency.

Noticing that $R_{x_{R}}^{ \pm 2 f_{c}}(\tau)=-R_{x_{I}}^{ \pm 2 f_{c}}(\tau)$, and replacing eq.(8) and eq.(9) in eq.(7) leads to the following expression for $\alpha \in$ $I_{x}$ :

$$
R_{x}^{\alpha}(\tau)=\frac{1}{2} \cos \left(\omega_{c} \tau\right) R_{s_{R}}^{\alpha}(\tau)+\frac{1}{2} \cos \left(\omega_{c} \tau\right) R_{s_{I}}^{\alpha}(\tau)
$$

In eq.(10), $x(t)$ is cyclostationary with $I_{x}=\left\{\frac{k}{T_{s}}, k \in \mathbb{Z}\right\}$. We notice that $I_{x}$ does not have any cyclic frequency depending on $f_{c}$, as expected in theory.

By definition, for any $\alpha \notin I_{x}, R_{x}^{\alpha}(\tau)=0$. This is also true for particular values of $\alpha= \pm 2 f_{c}$. But we also notice that $R_{x_{R}}^{ \pm 2 f_{c}}(\tau)=-R_{x_{I}}^{ \pm 2 f_{c}}(\tau) \neq 0$ which leads to:

$$
R_{x}^{ \pm 2 f_{c}}(\tau)=R_{x_{R}}^{ \pm 2 f_{c}}(\tau)+R_{x_{I}}^{ \pm 2 f_{c}}(\tau)=0
$$

This is the property we harness to develop our criterion: we expect that the estimation of $\hat{R}_{x}^{\alpha}$ will not converge in the same way to zero if $\alpha= \pm 2 f_{c}$ and $\alpha \notin I_{x_{R}}$. 


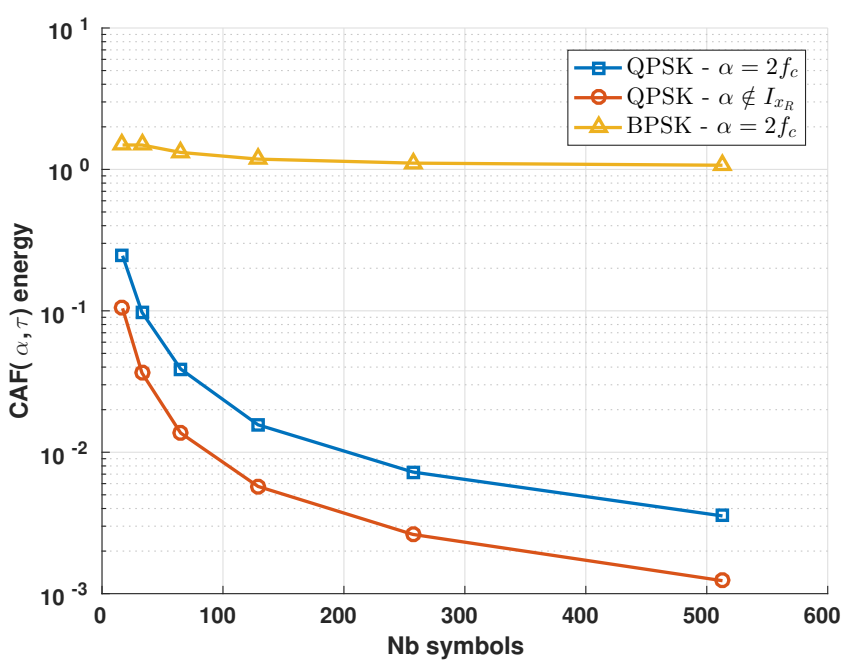

Fig. 1. CAF energy versus the observation duration (number of samples) for different modulation schemes (i.e. QPSK, BPSK) and for different $\alpha$

\section{Detection metric}

In this part, we present our criterion, defined after digitization. The theoretical CAF expression is given by eq.(6). However, in practice we only have one realization of the considered signal. Classically, to estimate the CAF, the expectation operator is replaced by a temporal average operator defined as $<.>=\frac{1}{N+1} \sum_{k=-N / 2}^{N / 2}$ (.). Rewriting eq.(6) with temporal averages leads to:

$$
\hat{R}_{x, N}^{\alpha}(l)=\frac{1}{N+1} \sum_{k=-N / 2}^{N / 2} x(k) x^{*}(k-l) e^{-j 2 \pi \alpha k}
$$

where the notation $\hat{R}_{x, N}^{\alpha}(l)$ stands for the estimate of $R_{x}^{\alpha}\left(l T_{s}\right)$, with $T_{s}$ the sampling period. When $N \rightarrow \infty$ eq.(12) meets the definition of the CAF defined in eq.(6).

By definition, for a given $\alpha \notin I_{x}, \hat{R}_{x}^{\alpha}(l) \triangleq 0$ since $\alpha$ is not a cyclic frequency. Applying the temporal average operation on eq.(11) leads to 0 only when $N \rightarrow \infty$ since $\lim _{N \rightarrow \infty} \hat{R}_{x_{R}}^{ \pm 2 f_{c}}(l)=\lim _{N \rightarrow \infty} \hat{R}_{x_{I}}^{ \pm 2 f_{c}}(l) \neq 0$. Consequently, with finite number of samples the behavior of the CAF estimator is different between the $\alpha= \pm 2 f_{c}$ and all the other non-cyclic frequency.

Our criterion $\hat{J}_{L, N}(\alpha)$ is defined as follows:

$$
\hat{J}_{L, N}\left(2 f_{c}\right)=\frac{\hat{C}_{N}^{2 f_{c}}(L)}{\hat{C}_{N}^{\beta}(L)}
$$

where $\beta \notin I_{x_{R}}$ and:

$$
\hat{C}_{N}^{\alpha}(L)=\frac{1}{L+1} \sum_{l=0}^{L}\left|\hat{R}_{x, N}^{\alpha}(l)\right|^{2}
$$

$L$ is the number of lags of the autocorrelation function considered, and $|$.$| is the modulus operator. As the$ precision of the estimated CAF in eq.(12) depends on $N$, so does eq.(14). This criterion can be interpreted as a reciprocal correlation coefficient (see [10]). Indeed, it makes a comparison between the estimated CAF at two $\alpha$ that are not cyclic frequencies. Our criterion can also be used for MPSK or MQAM modulations with $M>=2$. The only constraint is that $s_{R}(t)$ and $s_{I}(t)$ have to be independent.

The fig.1 illustrates the CAF speed convergence for different value of the cyclic frequencies $\alpha \in I_{x_{R}}$ and $\alpha \notin I_{x}$. Experimental conditions are detailed in the next section. In fig. 1 when $\alpha \in I_{x_{R}}$, the CAF estimator applied to a BSPK signal converges quickly through a non zero constant value (triangle yellow curve). In the same way, when $\alpha \notin I_{x_{R}}$, the estimator decreases in $1 / N$ and tends to zero (red circled curve). For a QPSK signal, when $\alpha=2 f_{c}$, the estimation speed convergence is similar. However, there is a gap between the curves $\alpha=2 f_{c}$ and $\alpha \notin I_{x}$.

Our criterion exploits this quasi-constant energy shift within those two curves and consequently, seems independent of $\mathrm{N}$. Another important remark is that even with a small number of symbols, we are able to differentiate the two cases considered above.

\section{DETECTION FEATURE ANALYSIS}

Let begin this section with a theoretical analysis of $\hat{J}_{L, N}(\alpha)$. We consider that the distribution of our criterion is obtained more easily in the absence of signal. Using the NeymanPearson approach [11], a threshold $\Gamma$ is determined with a constrained Probability of False alarm $P_{f a}=P\left[\hat{J}_{L, N}\left(2 f_{c}\right) \geqslant\right.$ $\left.\Gamma \mid H_{0}\right]$. The following statistical hypothesis test is defined below:

$$
H:\left\{\begin{array}{l}
H_{0} \rightarrow x(t)=n(t) \\
H_{1} \rightarrow x(t)=\Re\left[s(t) e^{i 2 \pi f_{c} t}\right]+n(t)
\end{array}\right.
$$

Here $H_{0}$ denotes the absence of signal and $H_{1}$ denotes its presence.

To define $\Gamma$, let us consider the $H_{0}$ hypothesis, where the numerator and the denominator of eq.(13) have same statistical properties. It was shown in [12], that the CAF estimator is unbiased and that error estimation is asymptotically complex normal. Re-expressing eq.(12) as the sum of a real and imaginary part leads to:

$$
\hat{R}_{x, N}^{\alpha}(l)=\Re\left[\hat{R}_{x, N}^{\alpha}(l)\right]+\jmath \Im\left[\hat{R}_{x, N}^{\alpha}(l)\right]
$$

where both real and complex terms follows asymptotically complex independent normal distributions for each $\tau$. The mean power expression in eq.(14) is consequently a sum of normal values, not reduced and non-centered. Under $H_{0}$, one can write the modulus expression of eq.(16):

$$
\left|\hat{R}_{x, N}^{\alpha}(l)\right|^{2}=\Re\left[\hat{R}_{x, N}^{\alpha}(l)\right]^{2}+\Im\left[\hat{R}_{x, N}^{\alpha}(l)\right]^{2}
$$




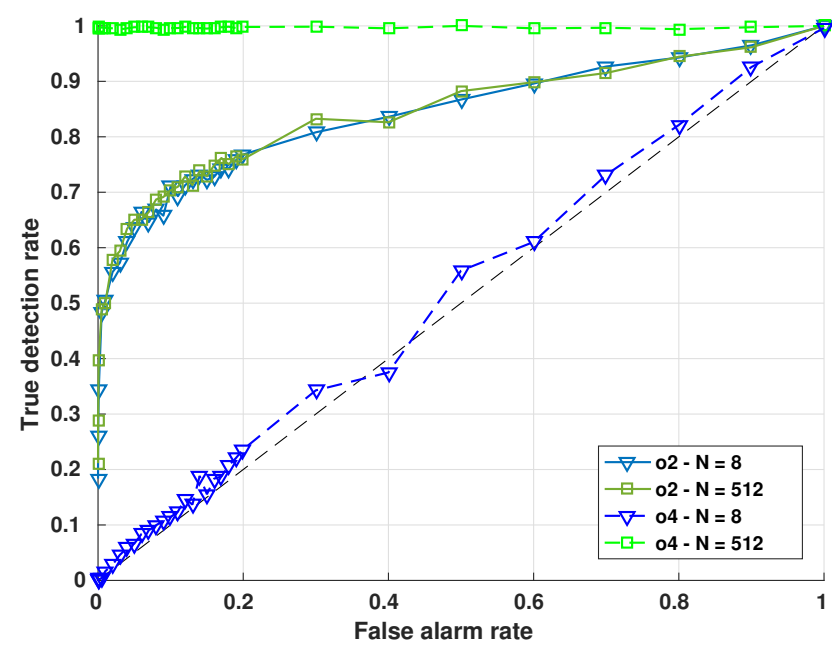

Fig. 2. ROC curves at fixed $\mathrm{SNR}=0 \mathrm{~dB}, 2048$ Monte-Carlo run, performance evolution for $N=8$ or $N=512$ number of symbols used

From eq.(14) and eq.(17) we get:

$$
(L+1) \hat{C}_{N}^{2 f_{c}}(L)=\sum_{l=0}^{L} \Re\left[\hat{R}_{x}^{2 f_{c}}(l)\right]^{2}+\sum_{l=0}^{L} \Im\left[\hat{R}_{x}^{2 f_{c}}(l)\right]^{2}
$$

Real and imaginary parts are independents and have same mean and variance.

Let now show that $\hat{J}_{L, N}\left(2 f_{c}\right)$ follows a doubly non-central Fisher distribution. Thanks to [11], we noticed that eq.(18) is quite similar to a non-central chi-2 distribution $\chi_{\nu}^{2}(\lambda)$, defined as:

$$
\chi_{\nu}^{2}(\lambda)=\sum_{i=0}^{\nu} \frac{X_{i}^{2}}{\sigma_{i}^{2}}
$$

Where $\lambda$ is the non-centrality parameter, $\nu=2(L+1)$ is the number of degrees of freedom and $X_{i}$ 's are independent and $X_{i} \sim \mathcal{N}\left(\mu_{i}, 1\right)$. However, eq.(18) is not a sum of reduced Normal distributions. We also notice that the doubly noncentral Fisher distribution is the ratio of two independent chi-2 distributions. Using eq.(19), $F_{\nu_{1}, \nu_{2}}^{\prime \prime}(\lambda, \delta)$ is denoted as:

$$
F_{\nu_{1}, \nu_{2}}^{\prime \prime}(\lambda, \delta)=\frac{\sum_{l=0}^{\nu_{1}} \frac{X_{l}^{2}}{\sigma_{l}^{2}}}{\sum_{k=0}^{\nu_{2}} \frac{X_{k}^{2}}{\sigma_{k}^{2}}}
$$

With our considered criterion, $\sigma_{l}^{2}=\sigma_{k}^{2}$ since we use eq.(14) to estimate $\hat{J}_{L, N}\left(2 f_{c}\right)$.

Consequently we proved that $(L+1) \hat{J}_{L, N}(\alpha) \sim F_{\nu_{1}, \nu_{2}}^{\prime \prime}(\lambda, \delta)$.

Non-centrality parameters $\lambda$ and $\delta$ tend to zero asymptotically, and the degrees of freedom $\nu_{1}=\nu_{2}=2(L+1)$. As eq.(13) is a ratio of random laws of similar moments, there is no need to determine $\lambda$ and $\delta$ since the ratio tends to 1 . At the asymptotic ( $\mathrm{N}$ is large) both $\hat{C}_{N}^{2 f_{c}}(L)$ and $\hat{C}_{N}^{\varepsilon}(L)$ tend to zero. Non-centrality

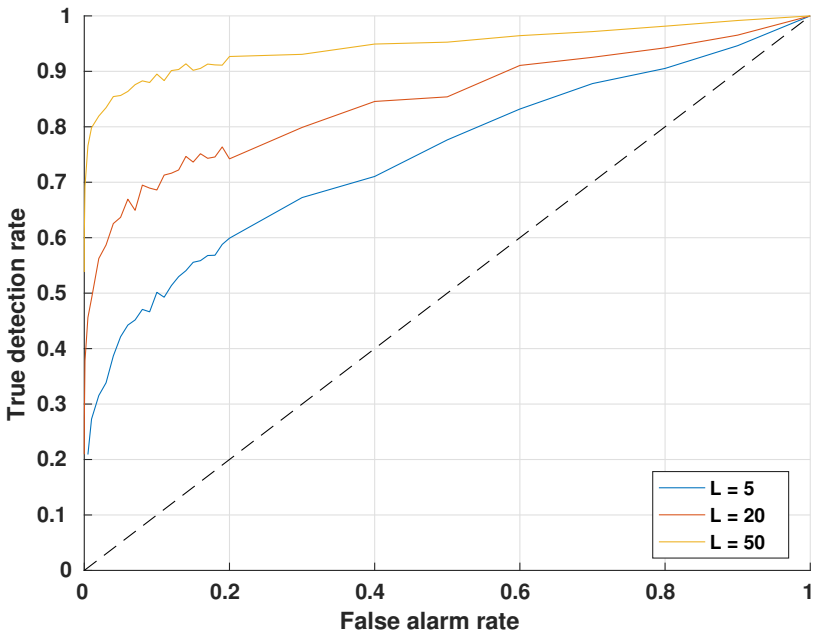

Fig. 3. ROC curves at fixed SNR $=0 \mathrm{~dB}, 2048$ Monte-Carlo run, fixed number of samples (64 symbols), performance evolution with several delays $M$

parameters become then $\lambda=\delta=0$. In that case eq.(13) follows a central Fisher law $F_{\nu_{1}, \nu_{2}}(x)$. This approximation works well in practice even for $N$ small. To fix $\Gamma$, the inverse function of $F_{\nu_{1}, \nu_{2}}(x)$ is required. A simple close form of $F_{\nu_{1}, \nu_{2}}(x)$ is detailed in [13]. However, it implies to use the inverse incomplete beta function, for which no close form expression exists. The usual method consists then in realizing an approximation. Thanks to the Newton algorithm and to the theoretical quantile function of central Fisher law, it becomes possible to determine a threshold $\Gamma$.

Before using the algorithm proposed below, the decision threshold has to be determined. However, as the SNR is unknown we have to fix a SNR value corresponding to a worst case of use. According to $H_{0}$, determine a threshold value such as for a given false alarm probability $P_{f a}=$ $P\left[F_{2(L+1), 2(L+1)} \geqslant \Gamma \mid H_{0}\right]$. Then, the same threshold will be applied for any SNR level. The proposed algorithm is the following one:

Step 1:Determine theoretical cyclic frequencies of the studied signal and set $\varepsilon$.

Step 2:Compute $\hat{R}_{x, N}^{2 f c}(l)$ and $\hat{R}_{x, N}^{\beta}(l)$ thanks to eq.(12) with $l \in[-L / 2, L / 2]$.

Step 3:Using eq.(14) and eq.(17), compute the test statistic $\hat{J}_{L, N}\left(2 f_{c}\right)$ in eq. (13).

Step 4:Decide $H_{1}$ if $J_{L, N}\left(2 f_{c}\right) \geqslant \Gamma$, and decide $H_{0}$ otherwise.

\section{Simulation RESUlTs}

\section{A. Experimental conditions}

In this part, we propose an analysis of the criterion defined in section II in order to show its accuracy for the considered application. We set a sampling rate $f_{s}=10 f_{c}$, in order to make a comparison with a high order moment detection 
method. As presented in introduction, in our considered application $f_{c}$ is the signal carrier frequency after down conversion. A square-root raised cosine filter of bandwidth $T=2.5 T_{c}$, is used as shaping filter with roll-of at 0.8 and span at 6 symbols. Monte Carlo simulations were run, in which we considered 2048 realizations to determine the false alarm probability $\left(H_{0}\right)$ and the power of the test $\left(H_{1}\right)$.

\section{B. Simulations}

As we show in previous sections, the criterion eq.(13) seems insensitive of $N$. In order to check this point, we fixed the SNR level to OdB. We set the maximum number of delays $L$ at 20. The ROC (Receiver Operating Characteristic) curves for $N=8$ and $N=512$ are presented in fig.2. We can see that our algorithm based on second order moments provides similar probability of detection with $N=8$ and $N=512$ (continuous lines). And we notice that the detector gives 65\% of good detection for a false alarm rate of $5 \%$. We compare those ROC curves with the ones obtained from the fourth order moment method (dashed lines) described in [7]. It is clear that this method outperforms our algorithm for $N=512$. But, we also noticed that for $N=8$, the probability of detection provided by the fourth order moment method dropped. This point is particularly interesting, since using our algorithm could significantly decrease the number of operation.

Let us now illustrate the influence of parameter $L$ on detection performance. The SNR level is fixed at $0 \mathrm{~dB}, N$ is fixed at 64 symbols. We plot ROC curves of several numbers of delay in fig.3. The most important result is that increasing $L$ provides a better rate of true detection. Considering only $L=5$ leads to poor detection performances. There is an improvement around $20 \%$ of true detection rate for the rise of $L$ from 5 to 20 delays. Then, increasing by 2.5 times the number of delays leads to another $20 \%$ rise of true

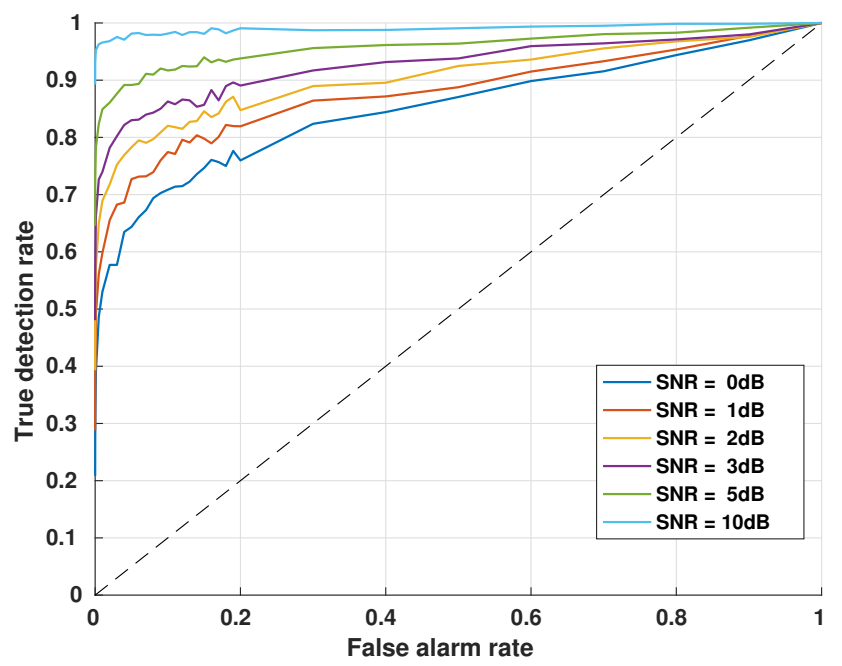

Fig. 4. ROC curves at number of samples (512 symbols), 2048 Monte-Carlo run, performance evolution for different SNR level

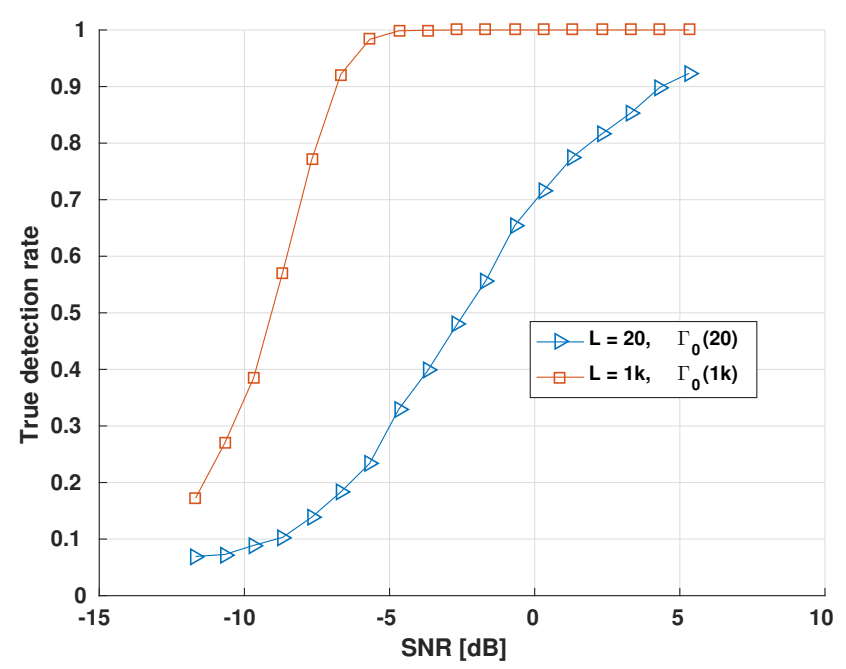

Fig. 5. Comparison of true detection rate for several delays L, with threshold $\Gamma$ defined at SNR fixed at $0 \mathrm{~dB}$

detection rate. Consequently, better performance are achieved by increasing the number of delay used in the criterion estimation. However, a trade-off between complexity and performance has to be made.

Another important question is the statistical test reliability with respect to the SNR. This is why we present ROC curves for several SNR levels in fig.4. We fixed $N$ at 512 symbols and $L$ at 20 delays. We can see a noticeable evolution of performance for the selected SNR levels. At SNR $=5 \mathrm{~dB}$, we reach a detection performance of almost $90 \%$ for a $5 \% P_{f a}$. The 10dB SNR obviously outperforms previous performance with more than $98 \%$ true detection at same false alarm probability.

Fig.5 illustrates the benefits to measure $\hat{J}_{L, N}(\alpha)$ with a large $L$ versus the SNR level. A threshold at $5 \% P_{f a}$ is estimated for several $L$ for each curve (20 and 1000). As we can see, the two curves decrease with the SNR level, to finally reach the $P_{t d}=5 \%$. This is in fact the $5 \%$ false alarm rate that we have defined. If we consider the $P_{t d}=70 \%$ at SNR $=$ $0 \mathrm{~dB}$ for $L=20$ as a reference, this performance is obtained at a SNR about $-8 \mathrm{~dB}$ for $L=1000$. In that case, $100 \%$ detection rate is also achieved for SNR $=-5 \mathrm{~dB}$. For SNR smaller than $-5 \mathrm{~dB}$, when $L$ is large, the $P_{t d}$ decreases also quickly. In fact, as $L$ increases, eq.(20) could be approximated by a Normal distribution. This approximation explains why the curve for $L=1000$ decreases quickly than the other one, since the Fisher distribution have a positive skewness when the Normal distribution is symmetric.

\section{Conclusion}

In this article, we proposed a novel method to detect phase modulated signals using a cyclostationarity analysis and low order moments. We show that it is possible to detect a 
signal presence based on second order moments at a cyclic frequency where there is theoretically no energy. We also discussed our method benefits and drawbacks and showed its limits. We derived the theoretical background and propose an algorithm. Finally, we showed through simulations that the proposed criterion is robust to noise. We also underlined that better performance are achieved for a large number of delays. The results obtained with the QPSK modulation are easily extended to phase modulated MPSK or MQAM (with $M>2$ ) signals. A major benefit of this method is that those measurements could be obtained for a small number of symbols. As prospects for future work, several studies could be conducted: a theoretical expression of the signal power estimator at $2 f_{c}$ has to be done, and a detection criterion improvement. For this last part, we propose to modify the criterion defined in eq.(13). The denominator would become a mean of $k$ values for a set of $\left\{\beta_{1}, \cdots, \beta_{k}\right\}$.

\section{ACKNOWLEDGMENT}

This work was conducted and funded in the scope of the FITNESS project (see [14]). The authors would like to thank all the FITNESS partners (Airbus Defense, MKRIC, BeSpoon, CEA, IMS, NXP, ISEP and ADS) for their cooperation.

\section{REFERENCES}

[1] T. Yucek and H. Arslan, "A survey of spectrum sensing algorithms for cognitive radio applications," Commun. Surveys Tuts., vol. 11, no. 1, pp. 116-130, Jan. 2009. [Online]. Available: http://dx.doi.org/10.1109/ SURV.2009.090109

[2] W. A. Gardner, "Signal interception: a unifying theoretical framework for feature detection," IEEE Transactions on Communications, vol. 36 , pp. 897-906, 1988.

[3] S. Houcke, "Séparation autodidacte d'une mélange de sources émettant à débits inconnus et éventuellement différents," 2002, Thesis.

[4] L. Tong, G. Xu, B. Hassibi, and T. Kailath, "Blind channel identification based on second-order statistics: a frequency-domain approach," IEEE Transactions on Information Theory, vol. 41, no. 1, pp. 329-334, Jan 1995.

[5] D. Boiteau and C. L. Martret, "A general maximum likelihood framework for modulation classification," in Acoustics, Speech and Signal Processing, 1998. Proceedings of the 1998 IEEE International Conference on, vol. 4, May 1998, pp. 2165-2168 vol.4.

[6] D. A. Hill and J. B. Bodie, "Carrier detection of psk signals," IEEE Transactions on Communications, vol. 49, no. 3, pp. 487-496, Mar 2001.

[7] J. Reichert, "Automatic classification of communication signals using higher order statistics," in Acoustics, Speech, and Signal Processing, 1992. ICASSP-92., 1992 IEEE International Conference on, vol. 5, Mar 1992, pp. 221-224 vol.5.

[8] C. M. Spooner, "On the utility of sixth-order cyclic cumulants for $\mathrm{rf}$ signal classification," in Conference Record of Thirty-Fifth Asilomar Conference on Signals, Systems and Computers (Cat.No.01CH37256), vol. 1, Nov 2001, pp. 890-897 vol.1.

[9] K. Kim, I. A. Akbar, K. K. Bae, J. S. Um, C. M. Spooner, and J. H. Reed, "Cyclostationary approaches to signal detection and classification in cognitive radio," in 2007 2nd IEEE International Symposium on New Frontiers in Dynamic Spectrum Access Networks, April 2007, pp. 212215.

[10] W. A. Gardner, Statistical spectral analysis, A non probabilistic theory. Englewood Cliffs,New Jersey: Prentice-Hall, 1988.

[11] S. M. Kay, Fundamentals of Statistical Signal Processing: Detection Theory. Pearson Education, 1998, vol. 2.

[12] A. V. Dandawate and G. B. Giannakis, "Statistical tests for presence of cyclostationarity," IEEE Transactions on Signal Processing, vol. 42, no. 9, pp. 2355-2369, Sep 1994
[13] N. Johnson and S. Kotz, Distributions in statistics: continuous multivariate distributions, ser. Wiley series in probability and mathematical statistics: Applied probability and statistics. Wiley, 1972.

[14] "Fitness scope," http://lisite.isep.fr/minarc/minarc-projects/fitness/, accessed: 2017-02-28 